\title{
CESIÓN CONTROLADA DE FÁRMACOS EN EL SEGMENTO POSTERIOR. NUEVAS TENDENCIAS
}

\author{
NEW TRENDS IN CONTROLLED DRUG DELIVERY TO \\ POSTERIOR SEGMENT
}

\author{
HERRERO VANRELL $\mathrm{R}^{1}$
}

Las patologías que afectan al segmento posterior del ojo se caracterizan por ser devastadoras y comprometer la visión en un gran número de sujetos. Dada la gravedad de estas enfermedades, resulta primordial tanto la instauración rápida del tratamiento como el mantenimiento de concentraciones eficaces, en el lugar de acción, durante el mayor tiempo posible. Sin embargo, la eficacia terapéutica de los tratamientos farmacológicos en esta zona se encuentra limitada, fundamentalmente, por la dificultad de acceso de la sustancia activa a los tejidos diana.

En la actualidad se utilizan cuatro tipos de administración encaminados a conseguir concentraciones eficaces en la zona posterior del ojo. Así, los fármacos se administran por vía tópica, sistémica, intraocular y periocular. Cuando se administra un fármaco por vía tópica en el momento de la administración se desencadenan distintos fenómenos, todos ellos encaminados hacia la eliminación del preparado de la zona córneal (se estima que menos de un 5\% de la dosis es capaz de llegar al interior del ojo) de forma que no se consiguen concentraciones eficaces en vítreo, retina y coroides. Si se recurre a la administración del fármaco por vía sistémica, las dosis necesarias para conseguir niveles terapéuticos en el lugar de acción son muy elevadas, generando, en muchos casos, efectos secundarios no deseados. El depósito directo del fármaco mediante inyecciones intravítreas es, dada la gravedad de la afección, una buena alternativa ya que, desde el primer momento, se consiguen concentraciones eficaces del principio activo en el vítreo. Las inyecciones intravítreas, sin embargo, llevan inherentes efectos secundarios como desprendimientos de retina, hemorragias, endoftalmitis y cataratas. Además, los tratamientos suelen requerir la administración de inyecciones repetidas, que no siempre resultan bien toleradas por el paciente. Con el fin de evitar administraciones frecuentes, se utilizan en la actualidad sistemas de cesión controlada de determinados principios activos adaptados a la administración. Dentro de los dispositivos intravítreos no biodegradables se encuentran dos implantes no biodegradables denominados Vitrasert, y Retisert ${ }^{\mathrm{TM}}$. El primero es de ganciclovir, y ha sido empleado para el tratamiento de la retinitis por citomegalovirus y el segundo de acetónido de fluocinolona para el tratamiento de la uveitis del segmento posterior. Los implantes, sin embargo, requieren cirugía y deben ser reemplazados periódicamente con el riesgo de producir efectos similares a aquellos asociados a las inyecciones intravítreas y producidos por la aparición de reacciones de rechazo.

En cualquier caso, ninguno de estos sistemas funciona a plena satisfacción y la dosificación de medicamentos hacía el segmento posterior del ojo esta demandando la búsqueda de nuevas rutas de administración menos agresivas que las empleadas hasta el momento actual así como el desarrollo de nuevas formulaciones que tengan propiedades más avanzadas que sus predecesoras.

En cuanto a la primera, la administración de fármacos por vía periocular ha cobrado un gran interés. El depósito de la sustancia activa en zonas adyacentes al lugar de acción como es el caso de la administración en los tejidos perioculares requiere que el fármaco atraviese la esclera (por simple difusión) para acceder al interior del ojo. Aunque puede haber pérdida de sustancia activa, las inyeccio-

\footnotetext{
1 Doctora en Farmacia. Profesora Titular. Departamento de Farmacia y Tecnología Farmacéutica. Facultad de Farmacia. Universidad Complutense. Madrid.

E-mail: rociohv@farm.ucm.es
} 
nes perioculares resultan menos agresivas y apuntan como alternativas ventajosas a las inyecciones intravítreas.

La administración del fármaco en solución hace que la duración de efectos sea corta ya que depende de la semivida de la sustancia activa. Por esta razón los investigadores se encuentran, desde hace años, diseñando e investigando nuevas formulaciones que den lugar a un efecto más prolongado disminuyendo, consecuentemente, el número de intervenciones. Dentro de los sistemas que se encuentran en periodo de evaluación destacan las nanopartículas $(1-1000 \mathrm{~nm})$ y las micropartículas $(1-1000 \mu \mathrm{m})$. Controlando la cesión de la sustancia activa, estos sistemas pueden dar lugar a cesiones más o menos prolongadas del fármaco. La ventaja principal de estas formulaciones estriba en que pueden ser administradas como una inyección convencional, pudiendo administrarse mediante inyecciones intraoculares y/o perioculares.

Si se recurre a un dispositivo de cesión controlada de la sustancia activa, la selección de los materiales formadores de los sistemas en estas formulaciones es vital ya que deben no sólo modular la cesión del fármaco sino ser altamente biocompatibles y exentos de toxicidad, cualidades que deben aplicarse también a los productos de degradación. Estos hechos reducen, considerablemente, el número de materiales empleados.

Desde hace treinta años, los polímeros derivados del ácido láctico y glicólico son uno de los biomateriales de mayor aplicación biomédica, fundamentalmente el copolímero derivado de los ácidos láctico y glicólico. Este hecho se basa en la excelente biocompatibilidad de estos polímeros biodegradables junto con la ausencia de toxicidad de sus productos de degradación. Las cadenas poliméricas son hidrolizadas para formar metabolitos naturales que se eliminan del organismo por el ciclo de Kreb's. Dependiendo de su composición y del peso molecular, estos polímeros pueden tener velocidades de degradación que van desde meses hasta años. Además, se puede seleccionar su aplicación en función de sus características. Por ejemplo, el ácido poliláctico de alto peso molecular se emplea como material de sutura cuando se necesita una elevada fuerza mecánica. Por el contrario, polímeros amorfos de bajo peso molecular son útiles en el desarrollo de sistemas de cesión controlada donde se pretende que el material desaparezca del lugar de acción una vez ejercido el efecto.
La búsqueda de nuevos materiales ha cobrado un gran interés en los últimos años. Se ha conseguido disponer de sustancias activas cuya actividad supera con creces a la de los fármacos habituales. Ya hay moléculas de gran actividad obtenidas por biotecnología y aprobadas por la FDA como es el caso de Lucentis ${ }^{\circledR}$ (ranibizumab), agente inhibidor del factor de crecimiento endotelial vascular (VEGF), destinado al tratamiento de la degeneración macular asociada a la edad (DMAE) exudativa. Los laboratorios, conscientes de la importancia de estos hallazgos, dan un paso más allá preocupándose no sólo de la protección de la nueva sustancia sino de la búsqueda de nuevos materiales cuyas características superen a los ya empleados y que a la vez puedan ser empleados en la elaboración de sistemas oftálmicos de cesión controlada. Así se están estudiando, entre otros, polímeros formadores de hidrogeles y polímeros sensibles a diferentes estímulos como la temperatura, determinados iones o $\mathrm{pH}$ del medio. Independientemente de su naturaleza a estos materiales se les exige gozar de unas características de biocompatiblidad óptimas y de propiedades que les permitan actuar como agentes moduladores de la cesión de la sustancia activa.

Hay mucho que decir y hacer en este campo. ¿Hacia dónde nos dirigimos? Este año, en ARVO 2007 (Association for Research in Vision and Ophthalmology), se realizará una evaluación de las técnicas actuales e innovativas para la cesión de sustancias activas en el segmento posterior durante una conferencia organizada por el Pfizer Ophthalmic Research Institute. Las técnicas evaluadas tendrán relación con el tratamiento de la degeneración macular asociada a la edad, edema macular en pacientes diabéticos, retinitis pigmentosa, neuroprotección de las células ganglionares en pacientes de glaucoma y el tratamiento de tumores oculares. Las presentaciones y las discusiones de dicha conferencia se publicarán en la revista Investigative Ophthalmology\& Visual Science. Esperemos la opinión de los expertos.

\section{BIBLIOGRAFÍA}

- Geroski DH, Edelhauser HF. Drug delivery for posterior segment eye disease. Invest Ophthalmol Vis Sci 2000; 41: 961-964.

- Wang N, Wu XS, Li C, Feng MF. Synthesis, characterization, biodegradation, and drug delivery application of biodegradable lactic/glycolic acid polymers: I. Synthesis and 
characterization. J Biomater Sci Polym Ed 2000; 11: 301 318.

- Ambati J, Canakis CS, Miller JW, Gragoudas ES, Edwards A, Weissgold DJ, et al. Diffusion of high molecular weight compounds through sclera. Invest Ophthalmol Vis Sci 2000; 41: 1181-1185.

- Herrero-Vanrel R, Refojo MF. Biodegradable microspheres for vitreoretinal drug delivery. Adv Drug Deliv Rev 2001; 52: 5-16.
- Lee SB, Geroski DH, Prausnitz MR, Edelhauser HF. Drug delivery through the sclera: effects of thickness, hydration, and sustained release systems. Exp Eye Res 2004; 78: 599-607.

- Kompella UB, Bandi N, Ayalasomayajula SP. Subconjunctival nano- and microparticles sustain retinal delivery of budesonide, a corticosteroid capable of inhibiting VEGF expression. Invest Ophthalmol Vis Sci 2003; 44: 1192-1201. 